\title{
COL4A1 mutation in an Indian child presenting as 'Cerebral Palsy’ mimic
}

\author{
Siddharth M Shah, Drushi D Patel ${ }^{1}$
}

Consultant Paediatric Neurologist, Royal Institute of Child Neurosciences, ${ }^{1}$ Consultant Radiologist, Gujarat Imaging Centre, Samved Hospital, Post Graduate Institute of Radiology and Imaging, Ahmedabad, Gujarat, India

Correspondence: Dr. Drushi D Patel, Gujarat Imaging Centre, Post Graduate Institute of Radiology and Imaging, Samved Hospital. 15t Floor, On H.L Commerce - Stadium Circle Road, Navrangpura, Ahmedabad - 380 009, Gujarat, India. E-mail: drushi43@yahoo.com

\section{Abstract}

The COL4A1 gene (COL4A1) plays an important role in vascular basement membrane function and pathogenic mutations have been reported in mice and humans. The gene is expressed mainly in the human brain, eyes and kidneys. Pathogenic mutations result in a vast array of manifestations that can present throughout life including the foetal period. We present a case of an 11-year-old girl with right hemiparesis, congenital cataracts, epilepsy and magnetic resonance imaging (MRI) brain findings with a pathogenic COL4A1 mutation. Many of her clinical features are similar to those of a non-genetic cause of cerebral palsy highlighting the difficulties and delays in making this genetic diagnosis.

Key words: Cerebral palsy; COL4A1 gene mutation; epilepsy; gliosis; magnetic resonance imaging; paediatric congenital cataract

\section{Introduction}

COL4A1 encodes for Collagen type 4 alpha 1, an important component of the hetero-trimers presents in nearly all vascular basement membranes ${ }^{[1]}$ It is particularly in the brain, muscles, eyes and kidneys. Most mutations are dominantly inherited and lead to structural instability of the affected basement membranes. These are missense mutations that replace $g$ glycine with another amino acid. A wide variety of presentations are described with mutations in COL4A1 including Hereditary angiopathy with neuropathy, aneurysms and muscle cramps (HANAC) due to mutations in exons 24 and 25 of the gene. ${ }^{[2]}$

The first report of a mutation in this gene was in mice in 2005, since then there have been reports of COL4A1 mutations causing intrauterine foetal cerebral haemorrhage

\begin{tabular}{|l|l|}
\hline \multicolumn{2}{|c|}{ Access this article online } \\
\hline Quick Response Code: & \\
\hline & Website: \\
\hline & www.ijri.org \\
\cline { 2 - 3 } & DOI: \\
\hline 口. & 10.4103/ijri.IJRI_274_20 \\
\hline
\end{tabular}

with secondary porencephaly; schizencephaly; and micro and macro haemorrhages with white matter changes noted in adulthood. ${ }^{[3,4]}$ In the paediatric age group, clinical presentations include epilepsy, developmental delay and problems with vision. Genetic studies have also demonstrated that manifestations can vary widely within affected families. ${ }^{[5-7]}$

\section{Case Report}

A 18-year-old girl initially presented to us when she was 11 years old. The pregnancy and delivery had been uneventful. Bilateral cataracts were noted after delivery and these were removed at 5 months of age. She started having focal seizures with secondary generalization at 6 months of age. Her development was delayed, and she was at a special

This is an open access journal, and articles are distributed under the terms of the Creative Commons Attribution-NonCommercial-ShareAlike 4.0 License, which allows others to remix, tweak, and build upon the work non-commercially, as long as appropriate credit is given and the new creations are licensed under the identical terms.

For reprints contact: WKHLRPMedknow_reprints@wolterskluwer.com

Cite this article as: Shah SM, Patel DD. COL4A1 mutation in an Indian child presenting as 'Cerebral Palsy' mimic. Indian J Radiol Imaging 2020;30:500-3.

Received: 18-Apr-2020

Accepted: $30-$ Oct-2020

Revised: 27-May-2020 Published: 13-Jan-2021 
needs school. Her parents and 11-year-old brother were in good health. There was no known family history of note including for stroke, cataracts and epilepsy or any extra CNS manifestations.

On examination, she had right hemiparesis. Her gross and fine motor difficulties did not deteriorate over time. She wore spectacles.

On brain MRI, she had volume loss with gliosis involving the left fronto-parietal periventricular white matter, centrum semi-ovale and ganglio-capsular regions with associated ex-vacuo dilatation of the left lateral ventricle. Confluent hyperintense signal was also noted involving the right fronto-parietal periventricular white matter and centrum semi-ovale on Fluid-attenuated Inversion Recovery (FLAIR) images suggestive of ischemic white matter changes. There was no evidence of acute ischemia or previous hemorrhage. Time-of-Flight) MR Angiography revealed paucity of insular and opercular branches of the left middle cerebral artery. There was no dolichoectasia or aneurysm formation [Figured 1-4].

Due to the absence of any peri-natal complications to explain the brain injury, and after investigations and excluding
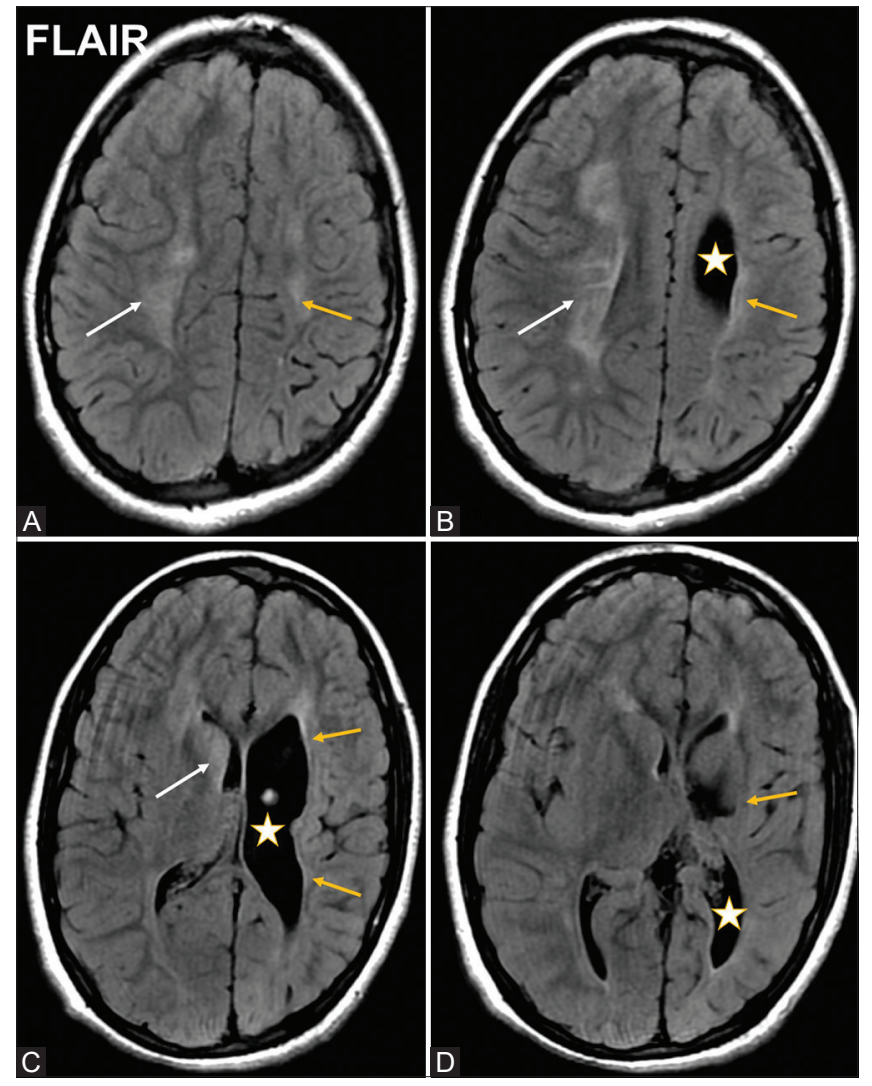

Figure 1 (A-D): Sequential FLAIR axial (A-D) MRI revealed gliosis with volume loss (yellow arrows) involving the left fronto-parietal periventricular white matter and ganglio-capsular regions with associated ex vacuo dilatation of left lateral ventricle (shown by star). Confluent hyperintense white matter ischemic changes (white arrows) were noted involving right fronto-parietal periventricular white matter other possibilities such as congenital infections, single gene testing of COLAA1 was requested. This revealed a novel c.2327G > T p.(Gly776Val) heterozygous mutation reported to be a likely pathogenic mutation. The parents have not been tested for the mutation.

\section{Discussion}

COL4A1 and COL4A2 located on chromosome13q34, encode for the collagen 4 alpha 1 and 2 chains respectively, and together form hetero-trimers that provide the supporting basement membrane of the vasculature of various tissues. Since the first description of mutations in mice leading to haemorrhage and porencephaly, and subsequent single and familial case reports, the spectrum of this disease has increased. The phenotype has grown not only in terms of possible CNS manifestations but also extra CNS manifestations. Subsequent to COL4A1 mutations, $C O L 4 A 2$ mutations have been reported to cause a milder but similar phenotype, and this not surprising as they are both important components of the heterotrimers. ${ }^{[8]}$ There have been reports of patients from all ethnicities and both genders. There have been reports from South East Asia including a few reports from India, ${ }^{[9]}$ however many cases remain underdiagnosed probably due to lack of recognition of these neurological presentations and lack of testing, as exemplified in our case in whom an initial diagnosis of a non-genetic form of cerebral palsy had been made.

Neurological presentations in childhood are usually the sequelae of porencephaly, schizencephaly, polymicrogyria, focal cortical dysplasia, and nodular heterotopia and associated white matter changes. ${ }^{[3-7]}$ Epileptic seizures reported include epileptic spasms, focal seizures as in our case, and generalized seizures. Adult case series reports indicate that the presentation tends to be that of a small vessel disease with ischemic and haemorrhagic infarction. Ocular phenotypes include congenital cataracts, microphthalmia, bilateral retinal arterial tortuosity, and anterior segment anomaly of the Axenfeld-Rieger type in which anterior chamber abnormalities comprising of congenital iris abnormalities, posterior embryotoxon,
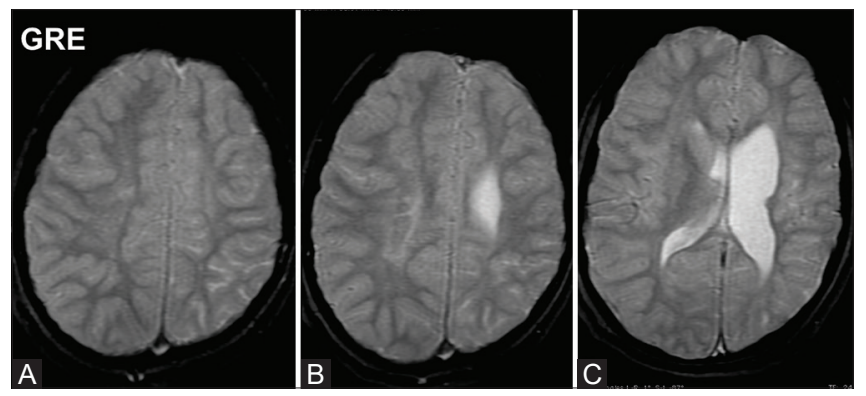

Figure 2 (A-C): (A-C): Sequential Gradient Echo (GRE) axial MRI showed no significant 'blooming' at the site of gliosis in left periventricular white matter and centrum semiovale to suggest hemorrhage or hemosiderin residue 
micro-cornea and glaucoma have been observed. ${ }^{[2]} \mathrm{A}$ recent large series reported patients with isolated congenital renal and urinary tract anomalies with non-glycine COL4A1 mutations. ${ }^{[9]}$

MRI findings ${ }^{[10-13]}$ tend to be nonspecific and include widespread confluent, bilateral, symmetric white matter T2 hyper-intensities with relative sparing of subcortical U-fibers and dilated perivascular spaces. There may be associated small lacunar infarcts predominantly involving the centrum semiovale, deep grey matter, or the brainstem. Cerebral micro-haemorrhage may be present particularly in the basal ganglia region because the microvasculature in these areas possesses sparse supporting tissue and the vessels branch at large angles. ${ }^{[14]}$ Van der Knapp et al. ${ }^{[15]}$ and Gould et al. ${ }^{[1]}$ have suggested that focal disruptions of the vascular basement membrane may predispose to haemorrhage and vessel wall abnormality, where as swelling of vascular endothelial cells and accumulation of fragmented basement membrane products may lead to narrowing of vessels and ischemic damage. No significant micro- or macro-hemorrhages were noted in our case.

If porencephalic cysts are noted these are often unilateral. ${ }^{[10]}$ In our case there was left sided gliosis with associated
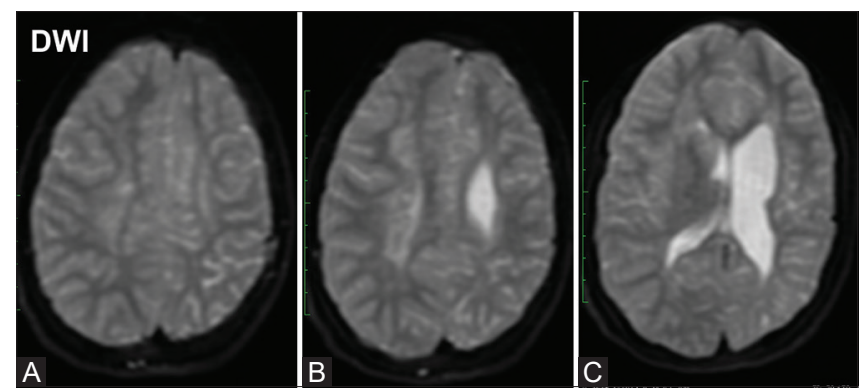

Figure $3(A-C)$ : $(A-C)$ : Sequential high 'b' value diffusion weighted (DWI) axial MRI did not show any abnormal hyperintense signal representing diffusion restriction involving the brain parenchyma to suggest acute ischemic changes ex-vacuo dilatation of left lateral ventricle. The differential diagnoses for such changes in the paediatric age group include mitochondrial diseases such as Mitochondrial Myopathy, Encephalopathy, Lactic Acidosis, Stroke-like episodes (MELAS), and central nervous system vasculitis especially when acute or in the chronic non-progressive form a prenatal or neonatal brain injury. In older individual's differential diagnoses include Cerebral Autosomal Dominant Arteriopathy with Subcortical Infarcts and Leukoencephalopathy (CADASIL), hypertensive microangiopathy, hereditary vascular retinopathy, cerebral amyloid angiopathy; Hereditary Endotheliopathy with Retinopathy, Nephropathy and Stroke (HERNS); Cerebral Autosomal Recessive Arteriopathy with Subcortical Infarcts and Leukoencephalopathy (CARASIL); and Fabry disease.

In our case, brain MRI changes and congenital cataracts were both present. In places with a very high prevalence of cerebral palsy, including India, similar static clinical findings and brain changes may mistakenly be attributed to brain injury due to perinatal events or congenital infections such as those caused by cytomegalovirus or toxoplasmosis. However, whilst strabismus and refractive errors have a high incidence in children with cerebral palsy, congenital cataracts are not a common occurrence and should lead to the consideration of other underlying diagnoses, especially in the absence of a clear neonatal history as in this case.

\section{Conclusion}

In summary, we present a case of a novel glycine COL4A1 mutation in an Indian patient presenting with bilateral congenital cataracts, epilepsy and abnormal brain MRI. The presence of associated ocular abnormalities such as congenital cataract, in the absence of a birth history that could lead to brain injury, should prompt consideration of a mutation in the COL4A1 gene leading to genetic testing. As genetic testing becomes reliable and more readily available, together with increasing awareness of such conditions, more
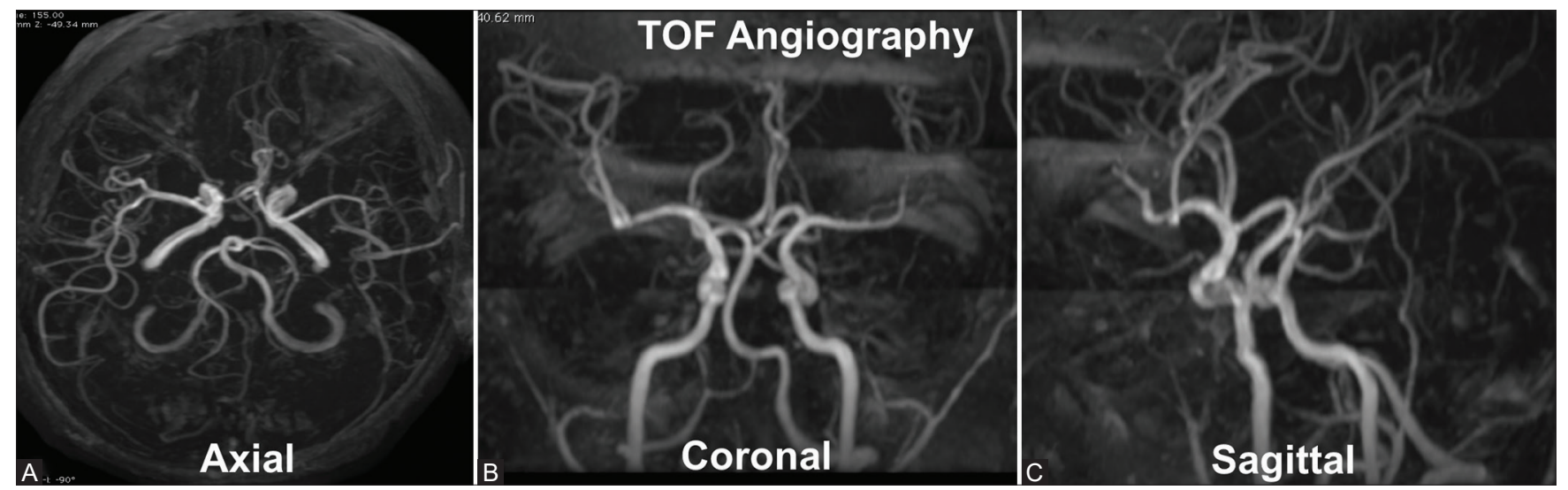

Figure 4 (A-C): (A-C): Axial (A), coronal (B) and Sagittal (C) 3D projections of TOF Angiography MRI reveals paucity of insular and opercular branches of left middle cerebral artery. No evident significant stenosis is noted involving visualized intracranial segment of internal carotid arteries, vertebro-basilar system and its branches. No evident aneurysm was noted 
cases will be confirmed and reported. Genetic counselling is very important as COLAA1 is an autosomal dominant disease with variable intra-familial presentation. Finally, as we begin to understand more about this gene and the resulting pathophysiological processes resulting from mutations within it, specific treatments initiated at an early stage may also become a realistic possibility in the future.

\section{Declaration of patient consent}

The authors certify that they have obtained all appropriate patient consent forms. In the form the patient(s) has/have given his/her/their consent for his/her/their images and other clinical information to be reported in the journal. The patients understand that their names and initials will not be published and due efforts will be made to conceal their identity, but anonymity cannot be guaranteed.

\section{Financial support and sponsorship}

Nil.

\section{Conflicts of interest}

There are no conflicts of interest.

\section{References}

1. Gould DB, Phalan FC, Breedveld GJ, van Mil SE, Smith RS, Schimenti JC, et al. Mutations in Col4a1 cause perinatal cerebral hemorrhage and porencephaly. Science 2005;308:1167-71.

2. Plaisier E, Gribouval O, Alamowitch S, Mougenot B, Prost C, Verpont MC, et al. COL4A1 mutations and hereditary angiopathy, nephropathy, aneurysms, and muscle cramps. N Engl J Med 2007;357:2687-95.

3. Yoneda Y, Haginoya K, Kato M, Osaka H, Yokochi K, Arai H, et al. Phenotypic spectrum of COL4A1 mutations: Porencephaly to schizencephaly. Ann Neurol 2013;73:48-57.

4. de Vries LS, Koopman C, Groenendaal F, Van Schooneveld M, Verheijen FW, Verbeek E, et al. COL4A1 mutation in two preterm siblings with antenatal onset of parenchymal hemorrhage. Ann Neurol 2009;65:12-8.

5. Shah S, Ellard S, Kneen R, Lim M, Osborne N, Rankin J, et al. Childhood presentation of COL4A1 mutations. Dev Med Child Neurol 2012;54:569-74.

6. Shah S, Kumar Y, McLean B, Churchill A, Stoodley N, Rankin J, et al. A dominantly inherited mutation in collagen IV A1 (COL4A1) causing childhood onset stroke without porencephaly. Eur J Paediatr Neurol 2010;14:182-7.

7. Zagaglia S, Selch C, Nisevic JR, Mei D, Michalak Z, Hernandez-Hernandez L, et al. Neurologic phenotypes associated with COL4A1/2 mutations: Expanding the spectrum of disease. Neurology 2018;91:e2078-88.

8. Yoneda Y, Haginoya K, Arai H, Yamaoka S, Tsurusaki Y, Doi H, et al. De novo and inherited mutations in COL4A2, encoding the type IV collagen $\alpha 2$ chain cause porencephaly. Am J Hum Genet 2012;90:86-90.

9. Nandeesh BN, Bindu PS, Narayanappa G, Yasha TC, Mahadevan A, Kulanthaivelu K, et al. Cerebral small vessel disease with hemorrhagic stroke related to COL4A1 mutation: A case report. Neuropathology 2020;40:93-8.

10. Kitzler TM, Schneider R, KohlS, Kolvenbach CM, Connaughton DM, Dai R, et al.COL4A1 mutations as a potential novel cause of autosomal dominant CAKUT in humans. Hum Genet 2019;138:1105-15.

11. Lanfranconi S, Markus HS. COL4A1 mutations as a monogenic cause of cerebral small vessel disease: A systematic review. Stroke 2010;41:e513-8

12. Vahedi K, Alamowitch S. Clinical spectrum of type IV collagen (COL4A1) mutations: A novel genetic multisystem disease. Curr Opin Neurol 24:63-8.

13. Renard D, Miné M, Pipiras E, Labauge P, Delahaye A, Benzacken B, et al. Cerebral small-vessel disease associated with COL4A1 and COL4A2 gene duplications. Neurology 2014;83:1029-31.

14. Lemmens R, Maugeri A, Niessen HW, Goris A, Tousseyn T, Demaerel $\mathrm{P}$, et al. Novel COL4A1 mutations cause cerebral small vessel disease by haploinsufficiency. Hum Mol Genet 2013;22:391-7.

15. van der Knaap MS, Smit LME, Barkhof F, Pijnenburg YA, Zweegman S, Niessen HW, et al. Neonatal porencephaly and adult stroke related to mutations in collagen IV A1. Ann Neurol 2006;59:504-11. 